\title{
Consolidation in short-term memory as a function of rehearsal'
}

\author{
MICHAEL J. A. HOWE \\ DALHOUSIE UNIVERSITY
}

In tests of short-term memory, intra-list differences have been observed in the extent to which accuracy of recall of items is affected by interfering events. No such differences occurred when all items were equally rehearsed, which suggests that the intra-list differences in resistance to interference were caused by differential rehearsal of items during presentation.

In a previous study (Howe, 1965) Ss listened to nine-consonant lists presented by ear at a rate of ore item per sec., and attempted recall of the sequences in various orders, specified after presentation. It was found that the items most accurately reproduced were those presented in the final three positions in a list, if recall of them preceded recall of the other items. However, the late items were the least accurately reproduced if they were the last items to be recalled. Conversely, items presented in the first three positions in a sequence were reproduced relatively inaccurately if they were the first to be recalled, but when reproduction of them was preceded by the activity of recalling the other items in the sequence, they were more accurately recalled than were items presented late in a sequence but in the corresponding recall position. Since recall commenced immediately after presentation of each list it was inferred that by the time presentation was complete, intralist differences existed in the extent to which storage of items was resistant to the distracting activity of recalling other items and/or to any other effects of the time spent in such activity. Consonants presented at the beginning of a sequence were more consolidated in memory than items occurring later in the same list.

By way of explanation, it is possible that during presentation of a list some rehearsal occurs, which would favor the early items, especially if Ss rehearse the consonants in sequence. Posner (1964) suggested that an interaction which he observed between effects of recall order and rate of presentation was due to the greater opportunity for rehearsal to occur during presentation at a slow than at a fast rate, and it has often been observed that allowing Ss to rehearse verbal material after presentation leads to greater accuracy in subsequent recall. However, it is possible that the degree of consolidation in verbal material stored over short periods is also related to the length of time over which the material has been retained, irrespective of the amount of active rehearsal. For instance, some involuntary circulation of stored sequences may occur.
The present experiment was carried out in order to determine whether consonants presented in early or late positions within verbal sequences differ over a short period in the degree to which they become consolidated in storage, when opportunities for rehearsal are equivalent for all items. Two groups of Ss differed in the extent to which they were free to structure rehearsal during presentation. For one group rehearsal was restricted to the immediately preceding letters, so that all items were equally rehearsed, whereas the other Ss were allowed to rehearse as they pleased. If rehearsal is the major determinant of intralist differences in short-term memory consolidation, the recall decrement produced by interpolating an interfering task between presentation and recall would not differ within lists for the group in which rehearsal was controlled, but there might be intralist differences in recall decrement for the group in which Ss' rehearsal during presentation was not constrained.

\section{Method}

The 60 Ss were undergraduates enrolled in undergraduate psychology courses at Dalhousie University, who participated as a course requirement. They were randomly assigned to two groups of 30 and were tested individually.

Lists containing the nine consonants CDFHLNRST, arranged in random orders, were presented on a memory drum. The letters appeared three at a time, for periods of 3 sec. and the interval between each three-letter group was less than $0.5 \mathrm{sec}$. Recall was required either immediately after presentation of the third three-letter group, or after an interfering task which succeeded the list. For the interfering tasks sequences of 14 digits, presented simultaneously, appeared for a duration of $3 \mathrm{sec}$. Ss had to commence reading the digits aloud, as fast as possible, and continue until the sequence disappeared. The interfering sequences occurred after half the consonant lists, specified at random.

A cue for recall immediately succeeded either the third, and final, three-item part of a list of letters, or the interfering sequence. Ss were required to recall just one of the three-letter groups in each list. Three horizontal black lines, typed side by side, appeared on the memory drum. Above one of them was typed another, parallel, line in red. A red line above the left hand black line indicated that recall was required of the letters on the card which had been presented first, i.e., consonants $1,2,3$, in the 
Tahle 1. Mean number of consonants correctly recalled by Aloud (A) and Silent (S) groups as a function of position in presentation sequence, in conditions of interference and no-interference between presentation and recall.

\begin{tabular}{llllll} 
& \multicolumn{5}{c}{ Positions of items in presentation sequence } \\
& & $1,2,3$ & $4,5,6$ & $7,8,9$ & Total \\
\hline $\begin{array}{l}\text { No interfering } \\
\text { task }\end{array}$ & A & 1.72 & 2.12 & 2.61 & 6.45 \\
& S & 1.76 & 1.98 & 2.32 & 6.06 \\
Interfering & A & 1.03 & 1.21 & 1.86 & 4.10 \\
task & S & 1.12 & 1.04 & 0.90 & 3.06 \\
Difference & A & 0.69 & 0.91 & 0.75 & 2.35 \\
& S & 0.64 & 0.94 & 1.42 & 2.90 \\
\hline
\end{tabular}

correct order. Lines above the middle and right hand lines indicated that recall was required of items $4,5,6$ and $7,8,9$ respectively.

Ss dictated their attempts at recall to E. Each $S$ attempted 48 lists, in 24 of which there was an interfering task between presentation and recall.

The recall cue occurred eight times within the first 24 lists and eight times within the remaining 24 lists at each of the three positions, and the order in which positions were used was random.

The two groups of Ss differed in the procedure which they were instructed to adopt during presentation of the lists. The first ("Aloud") group were told that as soon as three consonants appeared they were to commence reading the items out loud, as fast as possible, and to continue repeating them until they were replaced by other consonants, by the recall cue, or by an interfering sequence. The other ("Silent") group were simply told to attend to the consonants and attempt to remember them.

Eight practice lists preceded tue experiment. Letters recalled in the correct position were scored correct. Results

Table 1 shows mean number of consonants correctly recalled per three-item group as a function of the experimental variables. Mean recall of the Aloud group is more accurate than that of the silent group $(t=6.54, d f=58, p<.001)$ and the mean effect of the interpolated interfering task on recall is less for the Aloud than for the Silent group $(t=6.50, \mathrm{df}=58$, $\mathrm{p}<$.001).

In the Aloud group, the effect of the interfering task does not vary significantly between parts of lists $(\mathbf{F}=\mathbf{0 . 3 2})$. In the Silent group, however, there are considerable intralist differences in the mean decrement which occurs when a task is interpolated between presentation and recall $(t=7.29, d f=29, p<.001$ for the differences in mean recall decrements between items $1,2,3$ and $7,8,9$ and $t=4.71, \mathrm{df}=29, \mathrm{p}<.001$ between $4,5,6$ and $7,8,9$ ). This is consistent with the results obtained by the author (Howe, 1965) under conditions of auditory presentation.

Comparing groups for the intralist effects of the interfering task, it is found that the mean difference in recall decrement between items $7,8,9$ and $1,2,5$ is significantly greater in the Silent than in the Aloud group $(t=3.68, d f=58, p<.001)$.

\section{Discussion}

The results are consistent with the hypothesis that rehearsal during presentation is a major determinant of intralist differences in consolidation of consonants stored over short periods, since such differences do not occur when a procedure is used which ensures equal rehearsal of all items.

The observation that $S s$ who have to recite all items aloud perform better than Ss who do not have to do so is of interest, but the possibility cannot be excluded that the difference is due simply to the necessity for greater attention by the Aloud than by the Silent group, and hence may be unrelated to mechanisms of retention.

\section{References}

Howe, M. J. A. Intra-list differences in short-term memory. Quart. J. exp. Psychol., 1965, 17, 338-342.

Posner, M. I. Rate of presentation and order of recall in immediate memory. Brit. J. Psychol., 1964, 55, 303-306.

\section{Note}

1. The author is indebted to Barbara Candow who collected much of the data. 